\title{
Thermoelectric Transport through a Quantum Dot Coupled to a Normal Metal and BCS Superconductor
}

\author{
M. KRAWIEC \\ Institute of Physics, M. Curie-Skłodowska University \\ pl. M. Curie-Skłodowskiej 1, 20-031 Lublin, Poland
}

We discuss thermoelectric properties of a quantum dot coupled to one normal and one superconducting lead in the presence of the Kondo effect and the Andreev scattering. We will focus on conductance, thermal conductance, thermopower and related quantities like thermoelectric figure of merit which is a direct measure of the usefulness of the system for applications and the Wiedemann-Franz ratio which indicates if the system is in the Fermi liquid state. We will show that the superconductivity strongly modifies the thermal properties of the system. In particular, the thermopower is strongly enhanced near the superconducting transition temperature. Moreover, the Andreev reflections are suppressed due to strong on-dot Coulomb repulsion. The suppression of the Andreev reflections leads to a violation of the Wiedemann-Franz law and to a non-Fermi liquid ground state.

PACS numbers: 73.63.Kv, 73.23.-b, 73.50.Lw, 72.15.Qm, 74.45.+c

\section{Introduction}

Quantum dot (QD) devices provide a unique opportunity to study the interplay between different basic quantum phenomena. A prime example is the interaction of a localized spin (magnetic impurity) with surrounding itinerant electron spins [1]. This, known as the Kondo effect, manifests itself as a quasiparticle resonance in local density of states at the Fermi level at low temperatures. In quantum dots coupled to normal electrodes, it leads to an increase in conductance at zero source-drain voltage. Originally, the Kondo effect in quantum dots was predicted theoretically in the late 1980s $[2-4]$ and later demonstrated in a number of experiments [5-12].

Experiments have confirmed the validity of the theoretical picture but also discovered new phenomena. Those include observation of the Kondo resonance at non-zero source-drain voltage $[7,8]$, absence of even-odd parity effects expected for these systems [9], observation of singlet-triplet transition in magnetic field [10], splitting of the Kondo resonance due to the ferromagnetism in the leads [11] 
or interplay between magnetism (Kondo effect) and superconductivity in carbon nanotube quantum dots $[12,13]$.

When the quantum dot is connected to superconducting electrodes (S-QD-S), the low energy transport is mediated by the Andreev reflections, according to which an electron impinging on a normal metal-superconductor interface is reflected back as a hole and the Cooper pair is created in the superconductor [14]. This mechanism is known to play a crucial role in various hybrid mesoscopic superconducting devices [15]. In the S-QD-S system the Andreev transport is strongly affected by the Kondo effect, leading to the sign change of the Josephson current $[16,17]$. The strong Coulomb interaction prevents the tunneling of the Cooper pairs into QD, and the electrons tunnel via virtual processes. Thus the electron transport is strongly suppressed. This argument is valid when the Kondo temperature $T_{\mathrm{K}}$ is smaller than the superconducting order parameter $\Delta$. On the other hand, when $T_{\mathrm{K}}>\Delta$, the Kondo effect is restored [18]. This picture has been confirmed experimentally in carbon nanotube QD [12].

In the present work we consider a slightly different setup with a quantum dot coupled to one normal and one superconducting electrode (N-QD-S). This system has extensively been studied both theoretically [19-31] and experimentally [32]. Early theoretical works have predicted the enhancement of the conductance due to the Andreev reflections [21, 20], while the others have predicted the suppression of it $[19,22,23,26]$. Later on, it has been shown that the behavior of the Andreev conductance depends on the model parameters and can be enhanced or suppressed [23]. The experiment by Gräber et al. [32] shows that the conductance of $\mathrm{N}-\mathrm{QD}-\mathrm{S}$ system is suppressed in the Kondo regime.

The investigations of the $\mathrm{N}-\mathrm{QD}-\mathrm{S}$ systems have focused on electron transport only, and there is no study of thermoelectric properties. However, as is well known, the thermoelectric properties are the source of additional information to that obtained from other transport characteristics. Thermal properties (thermopower and thermal conductance) of strongly interacting quantum dot coupled to the normal and ferromagnetic leads have recently been investigated showing that thermopower is very sensitive and powerful tool to study the Kondo effect [33-39].

It is the purpose of the present work to study the thermoelectric properties of the quantum dot coupled to one normal and one superconducting electrode. We will focus on the electric and thermal conductance, thermopower and related quantities like thermoelectric figure of merit which is a direct measure of the usefulness of the system for applications and the Wiedemann-Franz ratio which indicates if the system is in the Fermi liquid state. We will show that the superconductivity strongly modifies the thermal properties of the system leading to an increase in thermopower at low temperatures and to non-Fermi liquid ground state.

Rest of the paper is organized as follows. In Sect. 2 we present the model and briefly discuss the approach. Results of calculations are presented and discussed in Sect. 3. Summary and conclusions are given in Sect. 4 . 


\section{Formulation of the problem}

The system is described by single impurity Anderson model with very strong on-dot Coulomb repulsion $(U \rightarrow \infty)$. In this limit there might be at most a single electron on the dot. To project out double occupied states it is convenient to introduce slave boson representation $[40,41]$, in which the real dot electron operator $d_{\sigma}$ is replaced by the product of boson $b$ and fermion $f_{\sigma}$ operators $\left(d_{\sigma}=b^{+} f_{\sigma}\right)$ subject to the constraint $b^{+} b+\sum_{\sigma} f_{\sigma}^{+} f_{\sigma}=1$. The resulting Hamiltonian reads

$$
\begin{aligned}
H= & \sum_{\lambda \boldsymbol{k} \sigma} \epsilon_{\lambda \boldsymbol{k}} c_{\lambda \boldsymbol{k} \sigma}^{+} c_{\lambda \boldsymbol{k} \sigma}+\sum_{\boldsymbol{k}}\left(\Delta c_{\mathrm{S} \boldsymbol{k} \uparrow}^{+} c_{S-\boldsymbol{k} \downarrow}^{+}+\text {h.c. }\right) \\
& +\varepsilon_{\mathrm{d}} \sum_{\sigma} f_{\sigma}^{+} f_{\sigma}+\sum_{\lambda \boldsymbol{k} \sigma}\left(V_{\lambda \boldsymbol{k}} c_{\lambda \boldsymbol{k} \sigma}^{+} b^{+} f_{\sigma}+\text { h.c. }\right)
\end{aligned}
$$

where $\lambda=\mathrm{N}(\mathrm{S})$ denotes normal (superconducting) electrode, $c_{\lambda \boldsymbol{k} \sigma}^{+}\left(c_{\lambda \boldsymbol{k} \sigma}\right)$ is the creation (annihilation) operator for a conducting electron with the wave vector $\mathbf{k}$, spin $\sigma$ in the lead $\lambda$, and $V_{\lambda \boldsymbol{k}}$ is the hybridization parameter between localized electron on the dot with energy $\varepsilon_{\mathrm{d}}$ and conducting electron of energy $\epsilon_{\lambda \boldsymbol{k}}$ in the lead $\lambda$. $\Delta$ is the superconducting order parameter in the lead $\mathrm{S}$, and its temperature dependence is chosen in the form: $\Delta(T)=\Delta_{0} \sqrt{1-\left(T / T_{\mathrm{c}}\right)^{2}}$ in superconducting state $\left(T \leq T_{\mathrm{c}}\right)$ and 0 otherwise.

In order to calculate electric current $J_{\mathrm{e}}$ and thermal flux $J_{Q}$ flowing from the normal electrode to the QD we follow standard derivation $[42,26]$ and get

$$
\begin{aligned}
J_{\mathrm{e}}= & J_{\mathrm{e}}^{\mathrm{sp}}+J_{\mathrm{e}}^{\mathrm{A}}=\frac{e}{h} \int \mathrm{d} \omega \mathcal{T}^{\mathrm{sp}}(\omega)[f(\omega-e V)-f(\omega)] \\
& +\frac{e}{h} \int \mathrm{d} \omega \mathcal{T}^{\mathrm{A}}(\omega)[f(\omega-e V)-f(\omega+e V)], \\
J_{Q}= & J_{Q}^{\mathrm{sp}}+J_{Q}^{\mathrm{A}}=\frac{1}{h} \int \mathrm{d} \omega \mathcal{T}^{\mathrm{sp}}(\omega)(\omega-e V)[f(\omega-e V)-f(\omega)] \\
& +\frac{1}{h} \int \mathrm{d} \omega \mathcal{T}^{\mathrm{A}}(\omega)(\omega-e V)[f(\omega-e V)-f(\omega+e V)],
\end{aligned}
$$

where the total current $J_{\mathrm{e}}$ and thermal flux $J_{Q}$ are divided into two parts, one associated with single particle processes (sp) and the other one corresponding to the Andreev reflections (A). As usual, $f(\omega)$ stands for the Fermi distribution function, and $e V=\mu_{\mathrm{N}}-\mu_{\mathrm{S}} \cdot \mathcal{T}^{\mathrm{sp}}(\omega)$ and $\mathcal{T}^{\mathrm{A}}(\omega)$ are the transmittances associated with single particle tunneling and with the Andreev reflections, respectively. The explicit form of $\mathcal{T}^{\operatorname{sp}(\mathrm{A})}(\omega)$ and the details of calculations can be found in Ref. [26].

In the linear regime, i.e. for small voltages $e V \rightarrow 0$ and small temperature gradients $\delta T=T_{\mathrm{N}}-T_{\mathrm{S}} \rightarrow 0$, one defines the electric conductance $G=-\frac{e^{2}}{T} L_{11}$, thermopower $S=-\frac{1}{e T} \frac{L_{12}}{L_{11}}$, and thermal conductance $\kappa=\frac{1}{T^{2}}\left(L_{22}-\frac{L_{12}^{2}}{L_{11}}\right)$. The kinetic coefficients read

$$
L_{11}=\frac{T}{h} \int \mathrm{d} \omega \mathcal{T}^{\text {tot }}(\omega)\left(\frac{\partial f(\omega)}{\partial \omega}\right)_{T},
$$




$$
\begin{aligned}
L_{12} & =\frac{T^{2}}{h} \int \mathrm{d} \omega \mathcal{T}^{\mathrm{tot}}(\omega)\left(\frac{\partial f(\omega)}{\partial T}\right)_{e V}, \\
L_{22} & =\frac{T^{2}}{h} \int \mathrm{d} \omega \mathcal{T}^{\mathrm{tot}}(\omega)(\omega-e V)\left(\frac{\partial f(\omega)}{\partial T}\right)_{e V},
\end{aligned}
$$

with equilibrium Fermi function $f(\omega)$ and total (single particle and Andreev) transmittance $\mathcal{T}^{\text {tot }}(\omega)$. In numerical calculations constant bands of width $100 \Gamma$ $\left(\Gamma=2 \pi\left(V_{\mathrm{S}}^{2}+V_{\mathrm{N}}^{2}\right)\right)$ have been assumed, and all the energies are measured in units of $\Gamma$.

\section{Results and discussion}

Figure 1 shows electric (left part) and thermal conductance (right part) for various values of superconducting order parameter $\Delta$. One observes strong suppression of the electric $(G)$ and thermal $(\kappa)$ conductance in superconducting state (for $T<T_{\mathrm{c}}=\frac{2 \Delta}{3.52}$ ). The reason for such a behavior is twofold. First, at $T=0$ the single particle transport is completely blocked due to the lack of the electron states in superconducting electrode for energies smaller than $\Delta$. Thus the only contribution to electric transport comes from the Andreev reflections. Let us note that the Andreev reflections do not contribute to the thermal transport (at $T=0$ ), as the electron is converted into hole, and thus there is no energy transfer through the interface. At the same time the Andreev scattering is only the mechanism which gives rise to electron transport (the charge of $2 e$ is transferred through the interface). However, strong Coulomb interactions on the dot prevent the tunneling of the Cooper pairs into QD, and the electrons in each pair can only tunnel one by one via virtual processes $[19,22,23,26]$. As a result, the Andreev tunneling is strongly suppressed. This is the second reason for such suppression of the electric conductance.

Corresponding linear thermopower $S$ is shown in Fig. 2. This quantity is very useful measure of the Kondo correlations [33-39]. At high tempera-
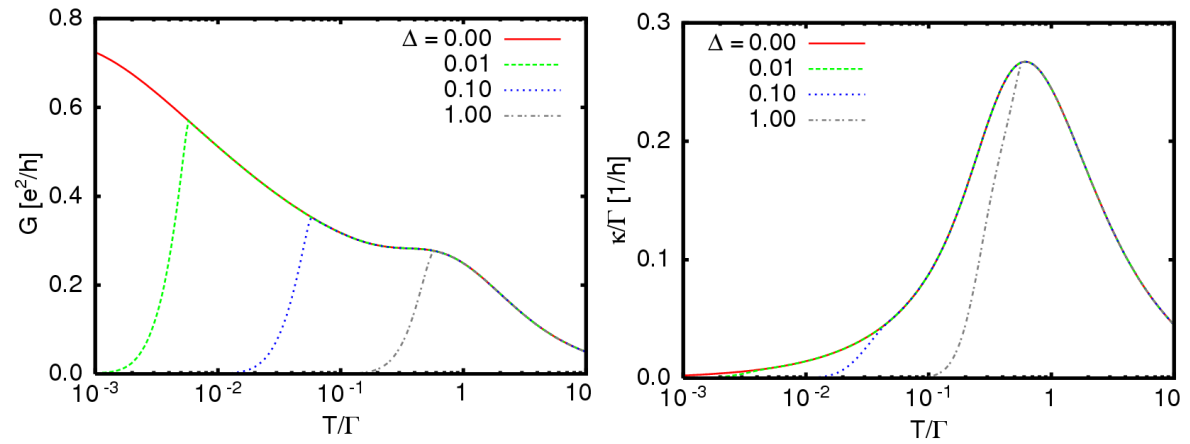

Fig. 1. Temperature dependence of electric (left part) and thermal conductance (right part) for various values of superconducting order parameter. The dot energy level $\varepsilon_{\mathrm{d}}=$ $-1.75 \Gamma$, and the Kondo temperature $T_{\mathrm{K}}(\Delta=0)=2 \times 10^{-2} \Gamma$. 


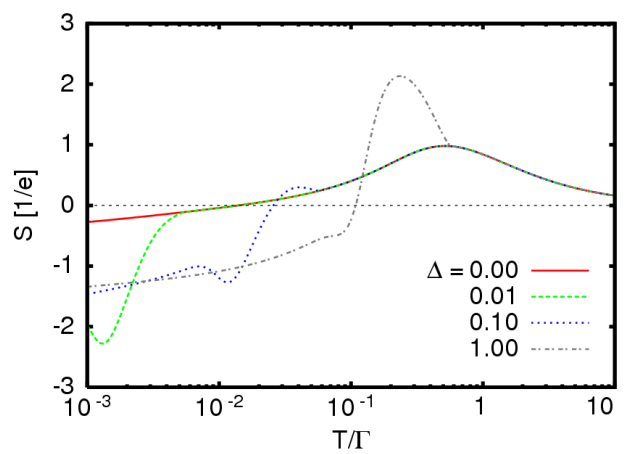

Fig. 2. Linear thermopower $S$ as a function of temperature for various values of superconducting order parameter $\Delta$.

tures thermopower is positive indicating hole-like transport and at $T \approx \Gamma$ shows a broad maximum associated with single particle excitations. At low $T$, where the transport is electron-like, the thermopower is negative. In the case of normal leads, the thermopower changes sign at the Kondo temperature $T_{\mathrm{K}}$ (solid line in Fig. 2). The sign change can be also understood from the fact that $S$ is sensitive to the slope of the density of states (DOS) at the Fermi level. With lowering of temperature, the Kondo correlations lead to the development of a narrow resonance in the DOS slightly above the Fermi energy, and thus to a slope change at the $E_{\mathrm{F}}$. In the presence of superconductivity in one of the leads, $S$ changes sign at superconducting transition temperature $T_{\mathrm{c}}$, rather than at the Kondo temperature. The sign change of $S$ and its behavior near $T_{\mathrm{c}}$ is associated with the shape of superconducting DOS, which is reflected in QD density of states.
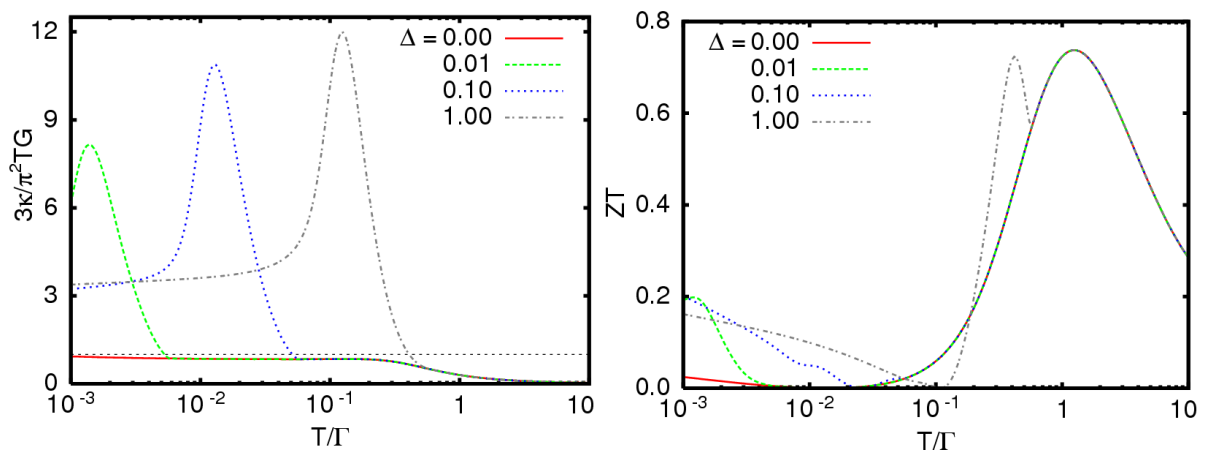

Fig. 3. The Wiedemann-Franz ratio $3 e^{2} / \pi^{2}(\kappa / T G)$ (left part) and thermoelectric figure of merit $Z T=S^{2} G T / \kappa$ (right part) as a function of temperature $T$.

Figure 3 (left part) shows the temperature dependence of the WiedemannFranz (WF) law which relates thermal and electric conductance via the relation $3 e^{2} / \pi^{2}(\kappa / T G)=1$. This relation describes transport in the Fermi liquid bulk 
metals, and usually is violated in nanoscale systems. However, below the Kondo temperature, the WF law is recovered, and the system has the Fermi liquid ground state $[33,37,39]$. In the high temperature regime, the WF law is violated as the transport is due to sequential processes leading to the suppression of the thermal transport [37]. This picture is valid for QD coupled to normal or to ferromagnetic electrodes. In the presence of superconductivity, this law is violated even at low temperatures (see left part of Fig. 3), indicating non-Fermi liquid ground state of the system. However, the superconductivity in one of the electrodes alone is not enough to violate the WF law. There must be strong Coulomb repulsion between electrons on the dot. In non-interacting QD $(U=0)$ the WF law is still obeyed at low $T$. The violation of the WF law in strongly interacting QD has its origin in suppression of the Andreev reflections, leading to smaller values of the linear conductance $G$ in comparison to non-interacting QD. At the same time, the Andreev reflections do not contribute to thermal transport, thus the thermal conductance remains unchanged. This explains the violation of the WF law in strongly interacting $\mathrm{N}-\mathrm{QD}-\mathrm{S}$ system.

Thermoelectric figure of merit $Z=S^{2} G / \kappa$ is a direct measure of the usefulness of the system for applications. For simple systems $Z$ is inversely proportional to operating temperature, therefore it is convenient to plot $Z T$. The right part of Fig. 3 shows $Z T$ as a function of temperature. The value of $Z T$ never exceeds 1 , which indicates limited applicability of the system for thermoelectric power generators or cooling systems. Let us note the enhancement of $Z T$ in superconducting state, which is due to the larger values of thermopower in this temperature range (see Fig. 2).

\section{Summary and conclusions}

In summary, we have studied thermal properties of strongly correlated quantum dot coupled to one normal and one superconducting electrode. The superconductivity strongly modifies thermal properties of the system. In particular it is responsible for an enhancement of thermopower at temperatures close to the superconducting transition temperature. The suppression of the Andreev reflections, which is due to strong on-dot Coulomb repulsion, leads to a violation of the Wiedemann-Franz relation and to non-Fermi liquid ground state.

\section{Acknowledgments}

This work was supported by the grant No. N N202 146833 of the Polish Ministry of Education and Science.

\section{References}

[1] A.C. Hewson, The Kondo Problem to Heavy Fermions, Cambridge University Press, Cambridge 1993.

[2] L.I. Glazman, M.E. Raikh, JETP Lett. 47, 452 (1988). 
[3] T.K. Ng, P.A. Lee, Phys. Rev. Lett. 61, 1768 (1988).

[4] A. Kawabata, J. Phys. Soc. Japan 60, 3222 (1991).

[5] D. Goldhaber-Gordon, H. Shtrikman, D. Mahalu, D. Abusch-Magder, U. Meirav, M.A. Kastner, Nature 391, 156 (1998).

[6] S.M. Cronenwett, T.H. Oosterkamp, L.P. Kouwenhoven, Science 281, 540 (1998).

[7] J. Schmid, J. Weis, K. Eberl, K. von Klitzing, Physica B 256-258, 182 (1998).

[8] F. Simmel, R.H. Blick, J.P. Kotthaus, W. Wegscheider, M. Bichler, Phys. Rev. Lett. 83, 804 (1999).

[9] J. Schmid, J. Weis, K. Eberl, K. von Klitzing, Phys. Rev. Lett. 84, 5824 (2000).

[10] S. Sasaki, S. De Franceschi, J.M. Elzerman, W.G. van der Wiel, M. Eto, S. Tarucha, L.P. Kouwenhoven, Nature 405, 764 (2000).

[11] A.N. Pasupathy, R.C. Bialczak, J. Martinek, J.E. Grose, L.A.K. Donev, P.L. McEuen, D.C. Ralph, Science 306, 85 (2004).

[12] M.R. Buitelaar, T. Nussbaumer, C. Schönenberger, Phys. Rev. Lett. 89, 256801 (2002); M.R. Buitelaar, W. Belzig, T. Nussbaumer, B. Babić, C. Bruder, C. Schönenberger, Phys. Rev. Lett. 91, 057005 (2003).

[13] A. Eichler, M. Weiss, S. Oberholzer, C. Schönenberger, A. Levy Yeyati, J.C. Cuevas, A. Martin-Rodero, Phys. Rev. Lett. 99, 126602 (2007).

[14] A.F. Andreev, Sov. Phys. JETP 19, 1228 (1964).

[15] C.J. Lambert, R. Raimondi, J. Phys., Condens. Matter 10, 901 (1998).

[16] M.-S. Choi, M. Lee, K. Kang, W. Belzig, Phys. Rev. B 70, 020502(R) (2004).

[17] G. Sellier, T. Kopp, J. Kroha, Y.S. Barash, Phys. Rev. B 72, 174502 (2005).

[18] Y. Avishai, A. Golub, A.D. Zaikin, Phys. Rev. B 67, 041301 (2002).

[19] R. Fazio, R. Raimondi, Phys. Rev. Lett. 80, 2913 (1998); Phys. Rev. Lett. 82, 4950 (1999).

[20] K. Kang, Phys. Rev. B 58, 9641 (1998).

[21] P. Schwab, R. Raimondi, Phys. Rev. B 59, 1637 (1999).

[22] A.A. Clerk, V. Ambegaokar, S. Hershfield, Phys. Rev. B 61, 3555 (2000).

[23] J.C. Cuevas, A. Levy Yeyati, A. Martin-Rodero, Phys. Rev. B 63, 094515 (2001).

[24] Y. Avishai, A. Golub, A.D. Zaikin, Phys. Rev. B 63, 134515 (2001).

[25] Q. Sun, H. Guo, T. Lin, Phys. Rev. Lett. 87, 176601 (2001).

[26] M. Krawiec, K.I. Wysokiński, Supercond. Sci. Technol. 17, 103 (2004).

[27] S.Y. Liu, X.L. Lei, Phys. Rev. B 70, 205339 (2004).

[28] Y. Tanaka, N. Kawakami, A. Oguri, J. Phys. Soc. Jpn. 76, 074701 (2007).

[29] A. Donabidowicz, T. Domański, Acta Phys. Pol. A 111, 671 (2007).

[30] T. Domański, A. Donabidowicz, K.I. Wysokiński, Phys. Rev. B 76, 104514 (2007).

[31] A. Donabidowicz, T. Domański, K.I. Wysokiński, Acta Phys. Pol. A 112, 157 (2007).

[32] M.R. Gräber, T. Nussbaumer, W. Belzig, C. Schönenberger, Nanotechnology 15, S479 (2004). 
[33] D. Boese, R. Fazio, Europhys. Lett. 56, 576 (2001).

[34] B. Dong, X.L. Lei, J. Phys., Condens. Matter 14, 11747 (2002).

[35] T.S. Kim, S. Hershfield, Phys. Rev. B 67, 165313 (2003).

[36] R. Scheibner, H. Buhmann, D. Reuter, M.N. Kiselev, L.W. Molenkamp, Phys. Rev. Lett. 95, 176602 (2005).

[37] M. Krawiec, K.I. Wysokiński, Phys. Rev. B 73, 075307 (2006).

[38] M. Krawiec, K.I. Wysokiński, Physica B 378-380, 933 (2006).

[39] M. Krawiec, K.I. Wysokiński, Phys. Rev. B 75, 155330 (2007).

[40] J.C. Le Guillou, E. Ragoucy, Phys. Rev. B 52, 2403 (1995).

[41] M. Krawiec, K.I. Wysokiński, Phys. Rev. B 59, 9500 (1999).

[42] H. Haug, A.P. Jauho, Quantum Kinetics in Transport and Optics of Semiconductors, Springer, Berlin 1996. 